\title{
PHOTOMETRY AND MODULATION OF LIGHT CURVES IN THE BLAZHKO STAR V365 HER
}

\author{
S.N. Udovichenko \\ Astronomical Observatory of I.I.Mechnikov Odessa National University \\ T.G.Shevchenko Park, Odessa 65014, Ukraine, udovich222@ukr.net
}

\begin{abstract}
The photometric CCD observations for the Blazhko RR Lyr type star V365 Her in Astronomical stations near Odessa (Ukraine) have been carried out. The light curves in $\mathrm{V}$ system were obtained and the frequency Fourier analyse was performed.The strong Blazhko effect and modulation of the light curves were detected. From Fourier spectra 15 frequencies were identified.
\end{abstract}

Key words: Stars: oscillations - stars; variables: RR Lyr - stars: individual: V365 Her.

\section{Introduction}

The variability of the star was found by C.Hoffmeister in 1936. The star thoroughly was investigated by V.P.Tsessevich, which determined the primary elements of period: Max. hel. J.D. = $2436047.522+0.6130535 \cdot \mathrm{E}$, and discovered the sophisticated shape of light variation curves, known as Bllazhko effect (40.64 day) (Tsessevich, 1961, 1966). He mentioned, that the curve of maximum height shows considerable dispersion, and it seems, that the most part of time we observe the weakened maxima and only in some time intervals (short-term) we observe the maxima in the considerable heights. It indicates in modulation of a light curves by additional frequencies, and the star represents the scientific interest for observations. Visual observations in 20 century were carried out by Hoffmeister and Tsessevich. Now V365 Her is known as RR Lyr-star type (RRab) with amplitude $12 .^{m} 7-14 .^{m} 0(\mathrm{P})$, has period $0 .^{d} 613138$, Kholopov et al. (1985).

\section{Observations}

The photometric CCD observations of V365 Her in Astronomical stations near Odessa in observation season 2009-15 have been carried out. Two stars were chosen as comparison and check stars (comp=UCAC4558-060233, check=UCAC4-558-060246). The $48 \mathrm{~cm}$ reflector AZT-3 with the $\mathrm{f} / 4.5$ Newtonian focus and CCD photometer with chip Sony ICX429ALL (600x800 pixels), equipped with $\mathrm{V}$ filter, Peltier cooler were used (Udovichenko, 2012). The hermetic housing and thermoelectric (Peltier) cooler provide a temperature difference between the crystal and the environment of about $-40^{\circ} \mathrm{C}$, and the temperature was supported by a constant. The exposure time for variable and comparison stars for the most part were chosen to except a saturation of frame and consist 90 sec. More then 5600 CCD frames were gathered during 55 nights. The reductions of the CCD frames were carried out using the MUNIPACK (Motl) software. The procedures for the aperture photometry are composed of the dark-level and flat-field corrections and determination of the instrumental magnitude and precision. The all set of observations are shown in fig.1. The errors on individual data points vary between 0.005 mag to 0.01 mag.

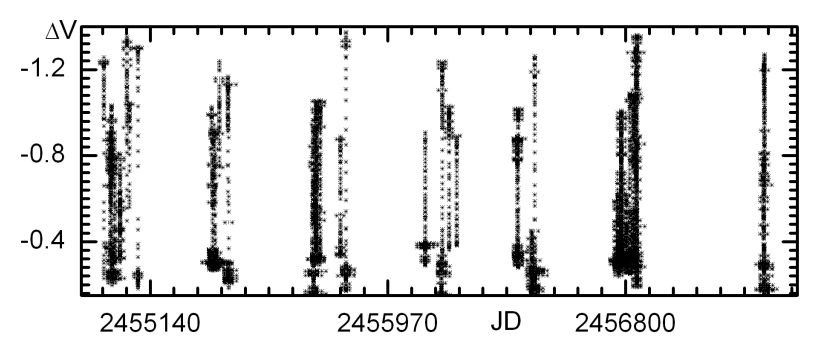

Figure 1: The all data set of observations V365 Her.

\section{Frequency analysis}

For all observations of V365 Her were determined the magnitudes comparatively of comparison star.

The frequency analyses were performed using a package of computer programs with single-frequency and multiple-frequency techniques by using utilize Fourier as well as multiple-least-squares algorithms (program Period04, Lenz and Breger, 2004). The pulsation period was determined with this package as the highest peak on the Fourier amplitude spectra. The all light curves V365 Her with pulsating period are shown on fig. 2. These phase curves were computed from elements:

$$
\text { MaxHJD }=2454978.403+0.613182 \cdot E .
$$


Table 1: Identified Fourier amplitude and phases of the pulsation and modulation frequencies in light curves of V365 Her.

\begin{tabular}{ccccc}
\hline Identif. & Frequency & Amplitude & Phase & $\mathrm{S} / \mathrm{N}$ \\
\hline f0 & 1.630835 & 0.346 & 0.15 & 6.6 \\
2f0 & 3.26168 & 0.127 & 0.86 & 26.7 \\
3f0 & 4.89255 & 0.086 & 0.89 & 20.2 \\
4f0 & 6.52330 & 0.061 & 0.22 & 13.1 \\
5f0 & 8.15355 & 0.052 & 0.15 & 11.7 \\
6f0 & 9.78498 & 0.021 & 0.43 & 6.1 \\
7f0 & 11.41402 & 0.021 & 0.68 & 4.6 \\
f0+fm & 1.65552 & 0.085 & 0.33 & 17.2 \\
f0- fm & 1.60613 & 0.054 & 0.54 & 11.7 \\
2f0-fm & 3.22425 & 0.034 & 0.74 & 6.6 \\
3f0+fm & 4.91979 & 0.034 & 0.83 & 7.1 \\
3f0-fm & 4.85806 & 0.026 & 0.85 & 7.0 \\
4f0+fm & 6.5530 & 0.034 & 0.26 & 7.6 \\
5f0+fm & 8.1788 & 0.038 & 0.80 & 6.7 \\
6f0-fm & 9.74763 & 0.024 & 0.47 & 6.1 \\
\hline
\end{tabular}

Table 2: Time of maxima V365 Her.

\begin{tabular}{ccc}
\hline Time J.D. & Time J.D. & Time J.D. \\
\hline 2454978.406 & 2455718.538 & 2456425.517 \\
2455000.510 & 2455720.377 & 2456785.478 \\
2455002.334 & 2455734.480 & 2456815.517 \\
2455005.391 & 2455804.382 & 2456828.398 \\
2455059.338 & 2455823.383 & 2456836.361 \\
2455067.319 & 2456159.387 & 2456839.427 \\
2455097.367 & 2456183.311 & 2457283.362 \\
2455355.527 & 2456210.284 & \\
2455411.336 & 2456422.466 & \\
\hline
\end{tabular}

The maximum of the light curves amplitude variation is $1.3 \mathrm{mag}$, minimum $0.8 \mathrm{mag}$, and strong Blazhko modulation of amplitude reach about $0.5 \mathrm{mag}$ and phase modulations up to 0.05 . The power spectra of basic frequency and after prewhitening is shown in Fig. 3.

After prewhitening of basic frequency the harmonics of basic frequency $\mathrm{kf0}$ and triplet of frequencies $\mathrm{kf}_{0} \pm \mathrm{f}_{m}$, responsible for modulation of light curves was found. The Fourier amplitude and phases of the pulsation component identified in the spectra of the light curves of V365 Her are presented in Table 1. The basic frequency denoted as $\mathrm{f}_{0}$, the modulation frequency of Blazhko effect denote as $\mathrm{f}_{m}$. We find triplet of frequencies $\mathrm{f}_{0} \pm \mathrm{f}_{B}$, but, perhaps, there are more frequencies in the pulsation spectra of V365 Her. From obtained data the mean period of Blazhko effect amount $14^{d} .6$.

The time of maxima V365 Her presents in Table 2.

\section{References}

Hoffmeister C.: 1936, Astron. Nachrichten., 259, 37. Lenz P., Breger M.: 2004, Comm.in Asteroseismology, 144, 41 .

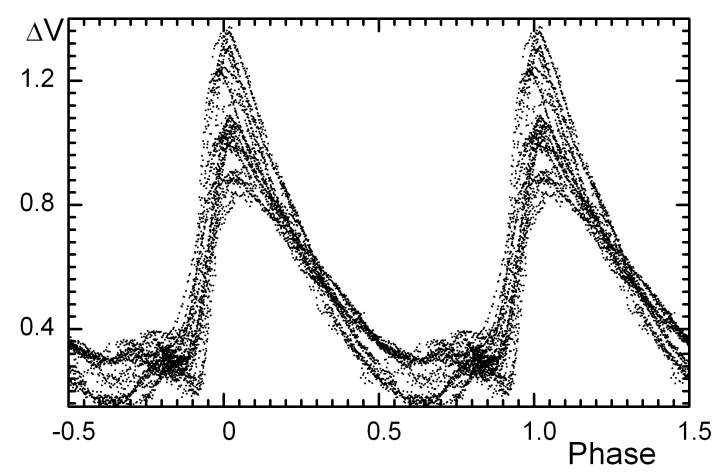

Figure 2: The light curves V365 Her with phase of basic period. The strong Blazhko modulation of amplitude reach about $0.5 \mathrm{mag}$ and phase modulations up to 0.05 .
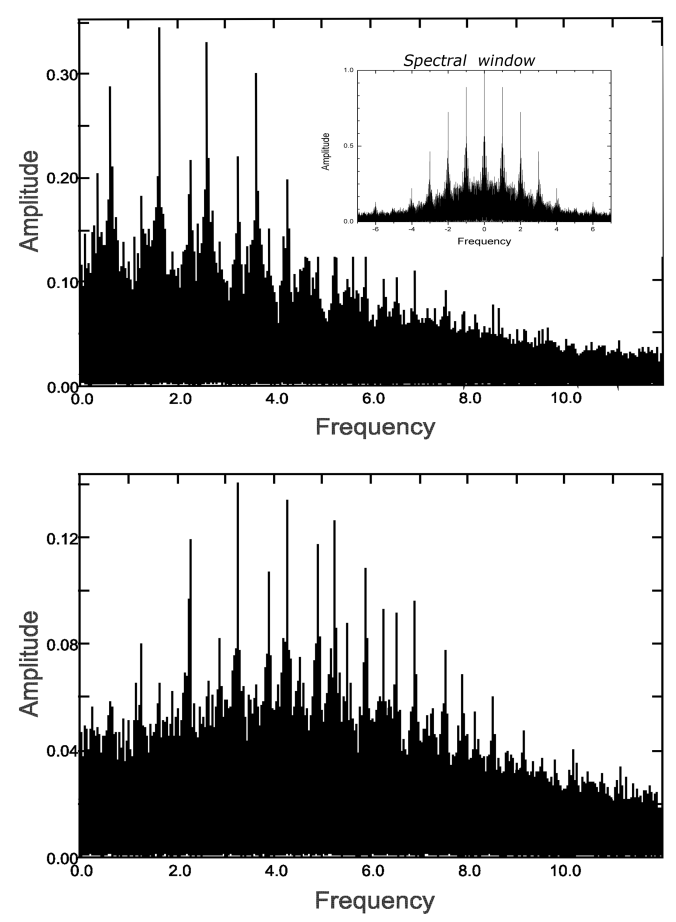

Figure 3: The Fourier amplitude spectrum of basic frequency and after prewhitening. The spectral window shows inside first picture.

Motl D.: http://sourceforge.net/projects/c-munipack/ files.

Samus N.N., Durlevich O.V., Kazarovets E.V., Kireeva N.N., Pastukhova E.N., Zharova A.V. et al.: 2011, General Catalogue of Variable Stars (GCVS database, Version 2011Jan).

Tsessevich V.P.: 1980, Sov. Astron.Jurn., 38, 293.

Tsessevich V.P.: 1966, RR Lyrae-type variable stars, Naukova Dumka, Kiev.

Udovichenko S.N.: 2012, Odessa Astron. Publ., 25, 32. 